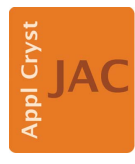

JOURNAL OF

APPLIED

CRYSTALLOGRAPHY

ISSN 1600-5767

Received 3 August 2015

Accepted 3 November 2015

Edited by A. J. Allen, National Institute of Standards and Technology, Gaithersburg, USA

Keywords: one-dimensional detectors; highresolution high-pressure powder X-ray diffraction; accurate intensities; powder diffraction; high pressure; synchrotron; structure solution; high resolution.

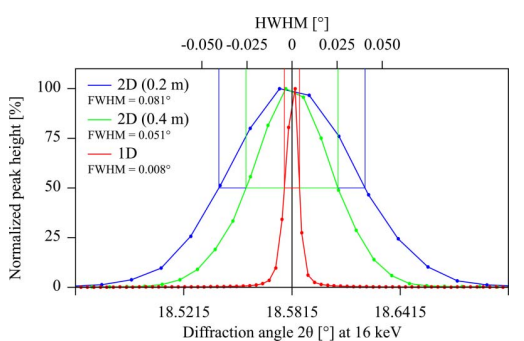

C 2015 International Union of Crystallography

\section{The benefits of one-dimensional detectors for high- pressure powder X-ray diffraction}

\author{
Martin Fisch, ${ }^{\mathrm{a}, \mathrm{b}}$ Arianna Lanza, ${ }^{\mathrm{a}, \mathrm{b}}$ Piero Macchi $^{\mathrm{a}}$ and Nicola Casati ${ }^{\mathrm{b} *}$ \\ ${ }^{\mathbf{a}}$ Department of Chemistry and Biochemistry, University of Bern, Freiestrasse 3, 3012 Bern, Switzerland, and ${ }^{\mathbf{b}}$ Swiss Light \\ Source, Paul Scherrer Institute, 5232 Villigen, Switzerland. *Correspondence e-mail: nicola.casati@psi.ch
}

High-pressure powder X-ray diffraction is a fundamental technique for investigating structural responses to externally applied force. Synchrotron sources and two-dimensional detectors are required. In contrast to this conventional setup, high-resolution beamlines equipped with one-dimensional detectors could offer much better resolved peaks but cannot deliver accurate structure factors because they only sample a small portion of the Debye rings, which are usually inhomogeneous and spotty because of the small amount of sample. In this study, a simple method to overcome this problem is presented and successfully applied to solving the structure of an L-serine polymorph from powder data. A comparison of the obtained high-resolution high-pressure data with conventional data shows that this technique, providing up to ten times better angular resolution, can be of advantage for indexing, for lattice parameter refinement, and even for structure refinement and solution in special cases.

\section{Introduction}

Increasing pressure on solid-state materials can provide a significant amount of energy to such systems, which is capable of altering nuclear positions, generating new phases and eventually triggering chemical reactions. Investigating materials' behaviour as a function of pressure is therefore essential to improve knowledge of structure-property relations and provides a basis for a deeper understanding of many condensed-matter phenomena.

With the invention of the diamond anvil cell (DAC; Jamieson et al., 1959), high-pressure (HP) studies have become widely accessible. Whereas DACs can be used in other analytical methods such as optical microscopy and spectroscopy, diffraction is the elective technique to obtain complete information on the crystal structure at a given pressure and temperature.

The high information content of single-crystal diffraction data makes it the method of choice for solving and refining structures at HP and such experiments can nowadays be routinely performed with laboratory X-ray diffractometers.

Often, however, HP experiments cannot be performed on single-crystalline samples, as they may not be available for a species or may experience severe fragmentation during firstorder pressure-driven phase transitions. Moreover, powder samples may be preferred when the phase evolution and transition points with increasing pressure are different for single crystals or powders, or in time-resolved investigations as experiments are faster (Boldyreva, 2007; Lanza et al., 2014; Fisch et al., 2015; Shekar \& Rajan, 2001; Perillat, 2008; Evans et al., 2007; Velisavljevic et al., 2014).

HP powder X-ray diffraction (P-XRD) experiments are, however, challenging and prone to many difficulties. Among 
the technical problems are e.g. absorption from the DAC, Compton scattering (leading to high background), very intense Bragg peaks from the diamonds, scattering from the metal gasket and the restricted reciprocal-space access due to shading from the DAC body. Moreover, at variance with traditional P-XRD experiments, random particle orientation, mandatory for extraction of reliable structure factors, is very difficult to achieve because of the very small amount of sample in a DAC (usually $\leq 200 \mu \mathrm{m}$ diameter and $\leq 80 \mu \mathrm{m}$ depth $=$ circa $0.003 \mathrm{~mm}^{3}$ ). In many cases, preferential orientation of the particles due to uniaxial stresses in the DAC (Tschauner $e t$ al., 2005) further affects the intrinsically weak and spotty diffraction. For data of acceptable quality, synchrotron radiation sources are usually required.

Even though the number of crystallites in the Bragg condition is increased when the DAC is oscillated a few degrees $\pm \omega$ (Smith \& Desgreniers, 2009), extraction of accurate intensities for structure solution or refinement from powder data still requires sampling of a large fraction of the Debye rings. Point or one-dimensional detectors are not suitable for such applications, and a typical setup involves a sample stage coupled with two-dimensional imaging plates, $\mathrm{CCD} / \mathrm{CMOS}$ detectors, or photon-counting pixel detectors with a high dynamic range to avoid saturation by high-intensity reflections from the sample and especially the diamonds. The frames are subsequently integrated and processed to a one-dimensional diffraction pattern with software such as the widely used Fit2D (Hammersley et al., 1996), GSAS-II (Toby \& von Dreele, 2013), XRD2DSCAN (Rodriguez-Navarro, 2006), Datasqueeze (Heiney, 2005) and various synchrotron inhouse developments, e.g. Dioptas (Prescher \& Prakapenka, 2015), Nika (Ilavsky, 2012) and DPDAK (Benecke et al., 2014), many of which are based on the Python library pyFAI (Ashiotis et al., 2015).

Two-dimensional detectors usually do not exceed a few megapixels, for practical and cost reasons. In order to collect the whole circumference of Debye rings up to small $d$ values, they have to be placed relatively close to the sample. Consequently, the spatial resolution of adjacent rings is reduced and peaks tend to overlap.

In summary, there is a constant trade-off between access to reciprocal space, angular resolution, and hence the accuracy of peak positions and extracted structure factors in HP P-XRD experiments.

In this study we discuss the use of a one-dimensional microstrip detector for this type of experiment, showing the advantages in resolution and describing a strategy for obtaining reliable intensities with such a setup. By comparing the obtained data with those measured with a two-dimensional detector, we show the potential of this method for applications which require extremely high resolution powder diffraction data at HP. This is showcased by investigating L-serine. An unsolved HP polymorph (L-Ser IV) was observed only in powder samples (Fisch et al., 2015; Boldyreva et al., 2006) and always coexisted with L-Ser I (ambient form) and L-Ser II (an already known HP form). The large cell of L-Ser IV results in severe peak overlap with the other phases. Indexing, space-
Table 1

Overview of the samples and measurement types.

\begin{tabular}{|c|c|c|c|c|}
\hline $\begin{array}{l}\text { Sample } \\
\text { name }\end{array}$ & Measurement type & Beamline & Compound & $\begin{array}{l}\text { Pressure } \\
(\mathrm{GPa})\end{array}$ \\
\hline SerA- $\chi$ & $\begin{array}{l}\text { Pseudo-two-dimensional } \\
\quad\left(360^{\circ} \chi\right)\end{array}$ & MS & L-Serine & $6.74(2)$ \\
\hline SerB-1D & $\begin{array}{l}\text { One-dimensional } \\
\quad \text { (one } \chi \text { position) }\end{array}$ & MS & L-Serine & $6.82(2)$ \\
\hline SerB-2D & $\begin{array}{l}\text { Two-dimensional } \\
\quad(0.2 \mathrm{~m} \text { detector distance })\end{array}$ & PXIII & L-Serine & $6.82(2)$ \\
\hline $\mathrm{LaB}_{6}-1 \mathrm{D}$ & $\begin{array}{l}\text { One-dimensional } \\
\quad \text { (one } \chi \text { position) }\end{array}$ & MS & $\mathrm{LaB}_{6}$ & 0.0001 \\
\hline $\mathrm{LaB}_{6}-2 \mathrm{D}$ & $\begin{array}{l}\text { Two-dimensional } \\
(0.2 \text { and } 0.4 \mathrm{~m})\end{array}$ & PXIII & $\mathrm{LaB}_{6}$ & 0.0001 \\
\hline
\end{tabular}

group assignment and extraction of structure factors for subsequent structure solution therefore profit from powder data with exceptionally high angular resolution.

\section{Experimental}

\subsection{Sample and DAC preparation}

Certified L-serine (Sigma-Aldrich 54763, lot No. $\mathrm{BCBH} 42640 \mathrm{~V}$ ) was ground in an agate mortar and further milled between finely ground glass plates to minimal particle size. Two samples (Table 1) were prepared in DACs with 0.5 and $0.6 \mathrm{~mm}$ culets. Steel discs with a hole of $0.25 \mathrm{~mm}$, preindented to circa $0.09 \mathrm{~mm}$, served as gaskets and a $4: 1$ $\mathrm{MeOH}: \mathrm{EtOH}$ solution was used as pressure medium. The pressure was raised with steps of <0.6 (1) GPa circa each $30 \mathrm{~min}$ as measured from ruby fluorescence (Syassen, 2008). The final pressure was determined from the L-serine lattice parameters (Fisch et al., 2015). Furthermore, a DAC with $0.3 \mathrm{~mm}$ culets was loaded with NIST SRM 660a $\mathrm{LaB}_{6}$ but not compressed (Table 1).

\subsection{Beamline setups}

Synchrotron powder XRD experiments were performed at the Swiss Light Source, Paul Scherrer Institute.

The X04SA Materials Science (MS) beamline's powder diffractometer (Willmott et al., 2013) is equipped with a PSI Mythen II one-dimensional single-photon-counting multistrip detector (Bergamaschi et al., 2010), mounted $0.76 \mathrm{~m}$ from the sample. It has a microstrip size of $50(\mathrm{H}) \times 800(\mathrm{~W}) \mu \mathrm{m}$, resulting in an intrinsic resolution smaller than $0.0038^{\circ} 2 \theta$. The curved detector covers a $2 \theta$ range of $120^{\circ}$ and was positioned to collect data from negative to positive diffraction angles. The radiation was monochromated to $16 \mathrm{keV}$ and the primary beam focused and shaped to a square of 100 by $100 \mu \mathrm{m}$. The wavelength and zero offsets were fine calibrated to the lattice parameters of NIST SRM 660a $\mathrm{LaB}_{6}$. A Huber Eulerian Cradle 511 (Huber Diffraktionstechnik GmbH \& Co. KG, Germany) was mounted onto the $\omega$ circle, allowing for $\chi$ rotation of the DAC coaxial with the beam. After centring the cradle to the beam, the gasket hole was aligned to the centre of the goniometer with a motorized $x y$ stage on the cradle's $\varphi$ circle. An overview of the setup is shown in Fig. 1. 


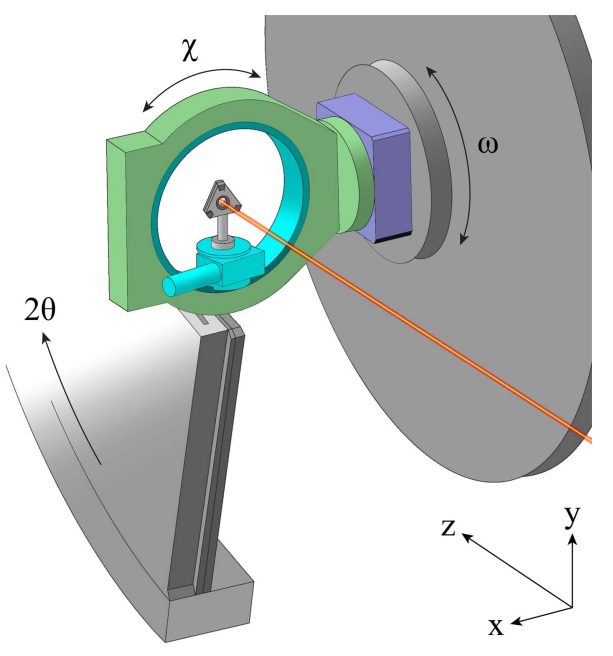

Figure 1

Illustration of the powder diffractometer setup at the X04SA MS beamline. The detector (not fully shown) covers both negative and positive diffraction angles. For alignment purposes, the DAC can be moved along $x$ and $y$ inside the Eulerian cradle. During data collection, $\chi$ is stationary and $\omega$ oscillates.

Two-dimensional diffraction data were collected at the X06DA Macromolecular Crystallography (PXIII) beamline (Waltersperger et al., 2015), equipped with a Dectris Pilatus $2 \mathrm{M}$ area detector (Broennimann et al., 2006). The detector and pixel size are $254(\mathrm{~W}) \times 289(\mathrm{H}) \mathrm{mm}$ and $172 \times 172 \mu \mathrm{m}$, respectively, and the beam $(16 \mathrm{keV})$ was focused and shaped to $90(\mathrm{~W}) \times 50(\mathrm{H}) \mu \mathrm{m}$. With the set detector distances of 0.2 and $0.4 \mathrm{~m}$, the angular coverage for complete Debye rings is $32.4^{\circ} 2 \theta$ and $17.6^{\circ} 2 \theta$, respectively. The best resolution for fully covered rings is calculated as $0.035^{\circ} 2 \theta$ at $0.2 \mathrm{~m}$ and $0.022^{\circ} 2 \theta$ at $0.4 \mathrm{~m}$ (Hinrichsen et al., 2008).

\subsection{Data collection}

Sample $\mathrm{LaB}_{6}-1 \mathrm{D}$ was measured at one fixed $\chi$ position with the one-dimensional detector and oscillated $\pm 3^{\circ} \omega$ during data collection. The same DAC $\left(\mathrm{LaB}_{6}-2 \mathrm{D}\right)$ was measured with the two-dimensional detector, with 0.2 and $0.4 \mathrm{~m}$ detector distances, and moved from $0^{\circ} \omega$ to $4^{\circ} \omega$ during acquisition.

All DACs loaded with L-serine (Table 1) were measured with the one-dimensional detector. In addition, sample SerB$2 \mathrm{D}$ was measured with the two-dimensional detector $(0.2 \mathrm{~m}$ distance) and the same $\omega$ rotation as the $\mathrm{LaB}_{6}$ DAC. The smaller detector distance ensures complete sampling of the Debye rings above $20^{\circ} 2 \theta$. A quadrant of the two-dimensional data is shown in Fig. 2.

On sample SerA- $\chi, 144$ diffraction patterns were collected with the one-dimensional detector each $2.5^{\circ} \chi$ while oscillating $\pm 6^{\circ} \omega$ during data acquisition. A quadrant of the pseudo-twodimensional image compiled from background-subtracted $\chi$ dependent raw data is shown in Fig. 2. Owing to the axial detector width of $8 \mathrm{~mm}$, overlap of the measured diffraction patterns (Debye ring completeness) decreases with increasing $2 \theta$, being larger than $100 \%$ up to $13.9^{\circ} 2 \theta$ and still $66 \%$ at $21^{\circ}$ $2 \theta$.

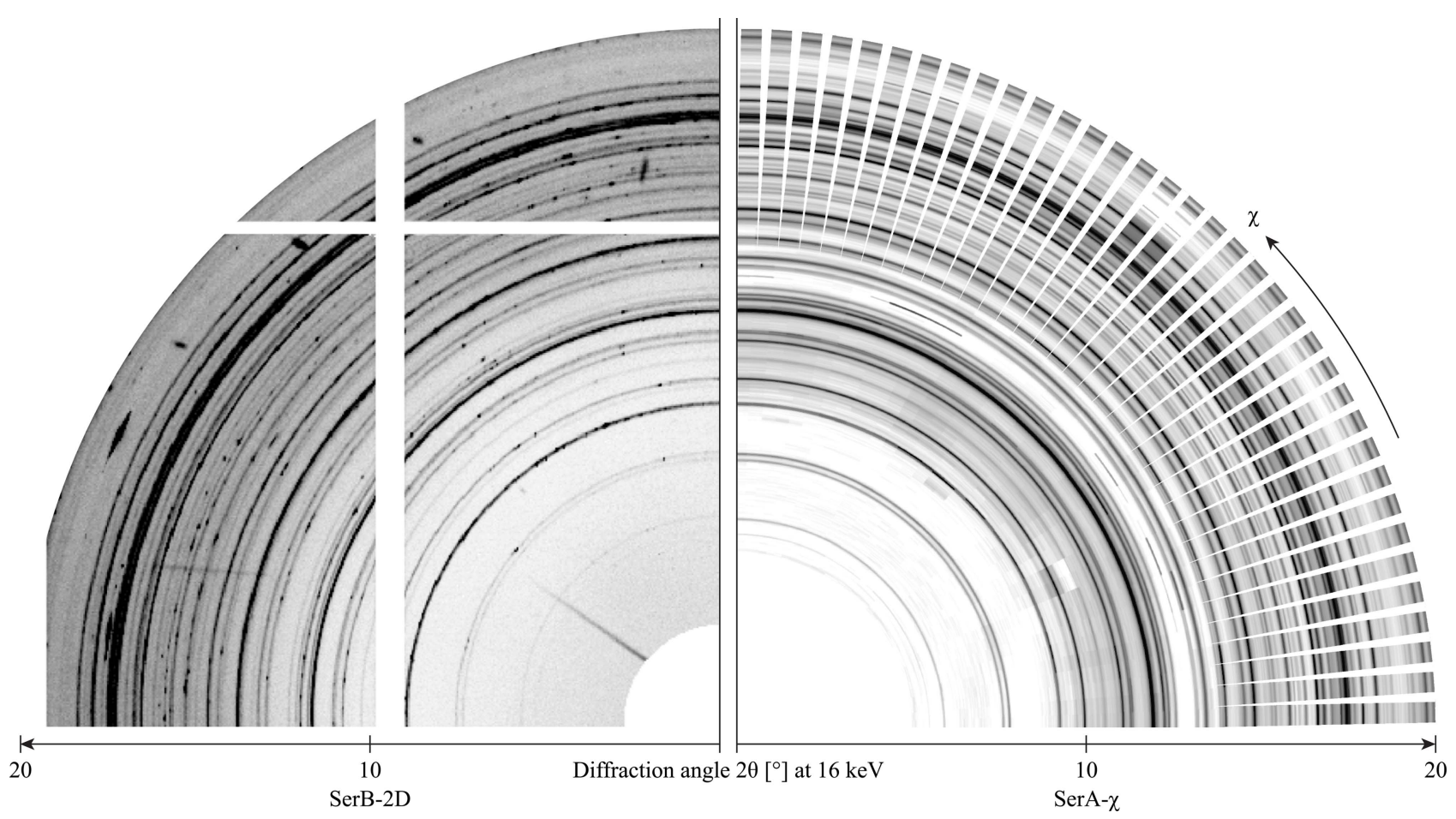

Figure 2

Left: the two-dimensional data of sample SerB-2D are shown from $0^{\circ} 2 \theta$ to $20^{\circ} 2 \theta$. The beam stop and gaps between detector modules are masked. Right: pseudo-two-dimensional visualization of the background-subtracted $\chi$-dependent raw data of sample SerA- $\chi$. Individual patterns from $5^{\circ} 2 \theta$ to $20^{\circ} 2 \theta$ are drawn as stripes on their corresponding $\chi$ angle. Above $13.9^{\circ} 2 \theta$, the sampling of the reciprocal space was not complete because of the chosen $\chi$ steps. These regions are therefore missing. 


\subsection{Data treatment}

$\mathrm{LaB}_{6}-2 \mathrm{D}$ frames were used to calibrate the integration parameters (detector centre, distance, tilt) of Dioptas version 0.2.4 (Prescher \& Prakapenka, 2015). All frames were subsequently integrated with Dioptas to .xy powder diffraction patterns with 2574 bins (equal to $0.0165^{\circ} 2 \theta$ per step at $0.2 \mathrm{~m}$ and $0.0098^{\circ} 2 \theta$ per step at $0.4 \mathrm{~m}$ ). Intensity s.u.'s were obtained with the SNBL Toolbox version $0.5 \sigma$-scaler (Dyadkin, 2015). The same frames were also integrated with Fit $2 D$ (Hammersley et al., 1996). The powder patterns matched those from Dioptas, except for slightly broader peaks, which most likely result from different data binning. Fit $2 D$ 's maximum bin width is limited to the smallest width of the detector (1475 bins), whereas Dioptas uses twice the longest distance from the Debye ring centre to the outmost pixel of the detector.

$\mathrm{LaB}_{6}-1 \mathrm{D}$ raw data were post-processed to an .xye diffraction pattern with a step size of $0.0036^{\circ} 2 \theta$ automatically after data acquisition.

All $\mathrm{LaB}_{6}$ patterns were Pawley refined using TOPAS Academic version 5 (Coelho, 2012) by applying a pseudoVoigt peak shape and the Caglioti equation (Caglioti et al., 1958) to determine the instrumental resolution function. The Caglioti parameter $U$ was set to 0 because of the restricted angular range ( irca $38^{\circ} 2 \theta$ before the DAC body shades the beam).

To obtain accurate L-Ser IV structure factors from the 144 $\chi$-dependent patterns of sample SerA- $\chi$, the following procedure was applied (Fig. 3). (1) From each pattern, all peak intensities were extracted with Pawley refined unit cells of L-Ser I, II and IV (intensities, background, damped lattice parameters, peak broadening). The cell of L-Ser IV was previously indexed with TOPAS by using the singular value decomposition algorithm (Coelho, 2003), yielding an orthorhombic cell with $P 2_{1} 2_{1} 2_{1}$ as the most probable space group, with $a=16.09873$ (5), $b=8.42773$ (6), $c=8.04459$ (7) $\AA$ and $V=$ $1091.46(2) \AA^{3}$. (2) The obtained structure factors were

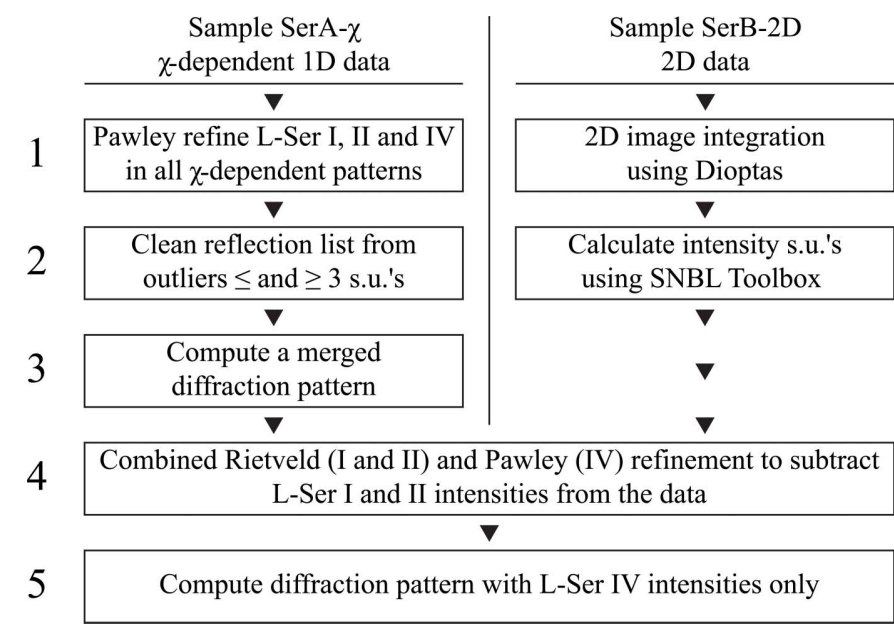

Figure 3

Data treatment procedure for extracting L-Ser IV structure factors from the diffraction patterns of samples SerA- $\chi$ and SerB-2D. cleaned from outliers $\leq$ and $\geq 3$ s.u.'s, and (3) a merged powder pattern was calculated using these average intensities. (4) This pattern was subsequently refined with a combined Rietveld (L-Ser I and II) Pawley (IV) approach in order to subtract L-Ser I and II intensities from overlapped peaks. Structural data of L-Ser I at 4.5 GPa were taken from Moggach et al. (2005) and those of L-Ser II at $6.3 \mathrm{GPa}$ from Wood et al. (2008). From the final Pawley intensities, (5) a powder pattern was computed and used to solve the structure.

Steps (4) and (5) were also applied to the data of sample SerB-2D in order to compare the intensities and structures obtained from the $\chi$-dependent data with those from the twodimensional data.

\subsection{Structure solution}

By starting from a supercell of L-Ser I with a tripled $a$ axis, the structure of IV was solved by simulated annealing (Coelho, 2000). Refined parameters included a scale factor, translation along the unit-cell axes (restricted to the three subcells in the $P 2_{1} 2_{1} 2_{1}$ supercell) and rotation of the three independent rigid-body molecules. The molecule geometry was taken from L-Ser I. Moreover, rotation of the $-\mathrm{CH}_{2} \mathrm{OH}$ and $-\mathrm{COO}$ groups about their corresponding $\mathrm{C}-\mathrm{C}$ axis (Fig. 4) was refined. $B_{\text {iso }}$ values were set to 0 . The resulting preliminary structure solution was used as the starting model for periodic density functional theory (DFT) calculations at the B3LYP level of theory using the full electron $6-31 \mathrm{G}(\mathrm{d}, \mathrm{p})$ basis set with CRYSTAL09 (Dovesi et al., 2009). The final structure $\left(R_{\text {Bragg }}=10.2 \%\right)$ was repeatedly obtained in several simulated annealing runs by using molecule geometries from the converged DFT calculation. This structure is discussed in detail by Fisch et al. (2015) and a CIF can be downloaded from the Cambridge Structural Database (CCDC number 1412832).

\section{Results and discussion}

From the DAC containing $\mathrm{LaB}_{6}$, the angular dependence of FWHM (Caglioti function parameters, Table 2) and peak shape of the two different setups was determined. The height-

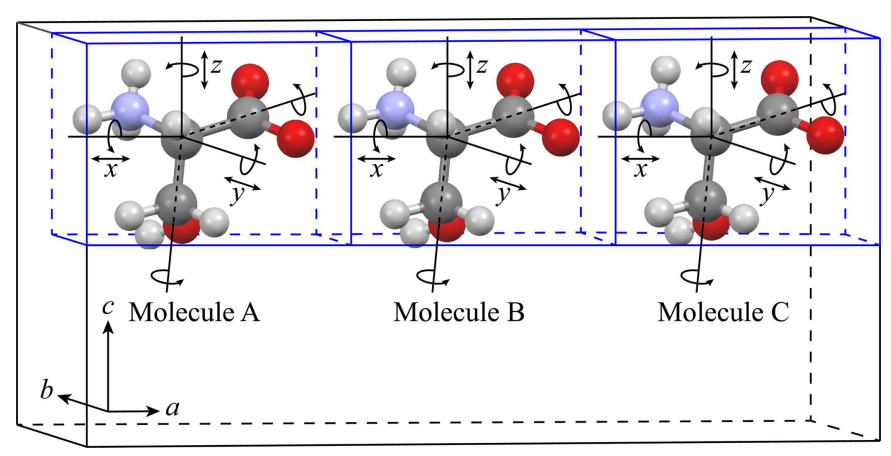

Figure 4

L-Ser I superstructure starting model. During simulated annealing, rotation and translation (restricted to the subcells of the asymmetric unit, blue boxes) of each molecule, as well as rotation of the $-\mathrm{CH}_{2} \mathrm{OH}$ and $-\mathrm{COO}$ groups around their corresponding $\mathrm{C}-\mathrm{C}$ axis, were refined. 
Table 2

Caglioti and peak shape parameters refined from the one-dimensional and two-dimensional $\mathrm{LaB}_{6}$ data sets.

\begin{tabular}{lllll}
\hline Data set & $W$ & $V$ & $U$ & $\eta \dagger$ \\
\hline $\mathrm{LaB}_{6}-2 \mathrm{D}(0.2 \mathrm{~m})$ & $0.00723(6)$ & $-0.0031(3)$ & 0 & 0 \\
$\mathrm{LaB}_{6}-2 \mathrm{D}(0.4 \mathrm{~m})$ & $0.00357(6)$ & $-0.0010(3)$ & 0 & 0 \\
$\mathrm{LaB}_{6}-1 \mathrm{D}$ & $0.000050(1)$ & $0.000173(6)$ & 0 & $0.271(2)$ \\
\hline
\end{tabular}

$\dagger$ Pseudo-Voigt mixing parameter $\eta=0$ for Gaussian profiles.

normalized 111 reflection of $\mathrm{LaB}_{6}$ of each data set is shown in Fig. 5.

$\mathrm{LaB}_{6}$ peaks measured with the one-dimensional detector are much sharper than those obtained from the two-dimensional data. The drastic difference in FWHM corresponds to a ratio of $10: 1$ for $0.2 \mathrm{~m}$ and still $6: 1$ for $0.4 \mathrm{~m}$ detector distance. The more than three times smaller pixel size of the curved Mythen II one-dimensional detector compared to that of the two-dimensional Pilatus $2 \mathrm{M}$ detector and almost twice the detector distance are the main reasons for the significantly higher resolution. Within the covered angular range, the FWHM of the one-dimensional data remains almost constant; however, slightly increasing peak broadening $(V>0$, Table 2) due to the sample, equatorial and axial divergence, and wavelength dispersion can be noticed, and the pseudo-Voigt peaks have circa $27 \%$ Lorentzian contribution. The FWHM of the two-dimensional data shows opposite behaviour $(V<0)$, which is expected because the flat detector lies tangentially on the goniometer radius. X-rays diffracted at high diffraction angles impinge more tilted on the detector than at low angle and are therefore better resolved (Hinrichsen et al., 2008). This effect is stronger than any other effect (e.g. sample broadening), and in sum, the FWHM measured from $\mathrm{LaB}_{6}$ slightly decreases with increasing $2 \theta$.

In practice, the absolute intensities of the two setups are not comparable. Among the reasons are differences in primary beam intensities, detector response and post-processing, absorption in air, and irradiated sample volumes. Identifica-

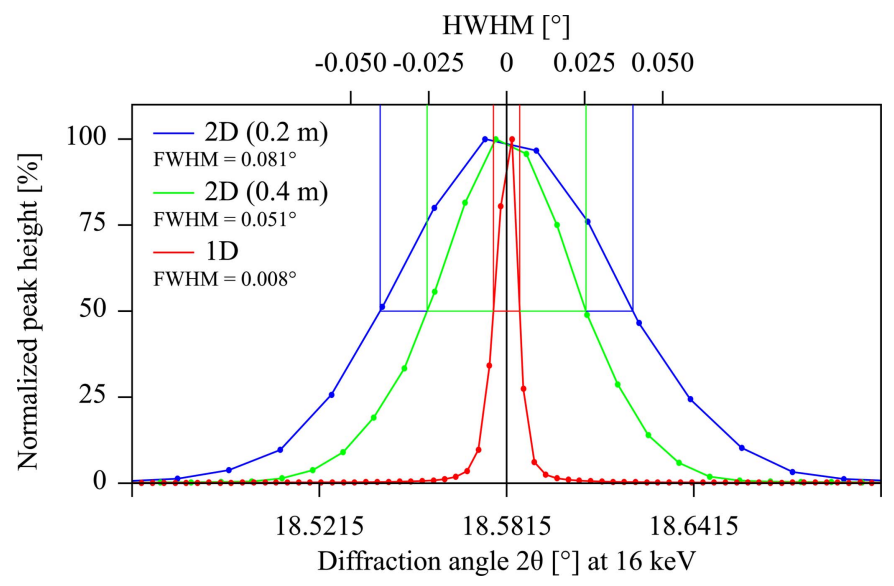

Figure 5

Raw data of the $\mathrm{LaB}_{6} 111$ reflection with normalized peak height. The secondary $x$ axis indicates half-width at half-maximum (HWHM) of the peaks. tion of the sharper peaks measured with the one-dimensional detector is nonetheless more straightforward, also because they have a larger maximum height compared to broader ones with the same integrated area. Consequently, they are better resolved from their baseline.

The smaller FWHM is advantageous for the precision of peak position determination. As an example, the uncertainty of Pawley refined $\mathrm{LaB}_{6}$ lattice parameters is more than three times smaller in the one-dimensional than in the two-dimensional data. The obtained values are 4.1569160 (16) and 4.1569165 (59) $\AA$ for the one-dimensional and the twodimensional data at $0.2 \mathrm{~m}$, respectively, when refined from the same ten reflections.

Sample SerB-1D was measured at one fixed $\chi$ position, as well as with the two-dimensional detector (sample SerB-2D) at $0.2 \mathrm{~m}$ detector distance. In the latter data, reflections of the pressure calibrant ruby sphere contribute to the diffraction pattern, whereas no ruby diffraction is observed in the onedimensional data. Enlarged regions of Pawley refined diffraction patterns obtained from both setups are shown in Fig. 6. The effect of the superior angular resolution of the onedimensional data becomes instantly noticeable. For example, the first two reflections of L-Ser II at $9.21^{\circ} 2 \theta$ and $9.25^{\circ} 2 \theta$ ('A', Fig. 6) are well separated in the one-dimensional data, whereas only a broad superimposed peak is observed in the two-dimensional data. The same is true for the reflection of $\mathrm{L}-$ Ser I at $10.53^{\circ} 2 \theta$ and that of IV at $10.57^{\circ} 2 \theta$ ('B'). Moreover, the intensity extraction of the sharper peaks of the one-

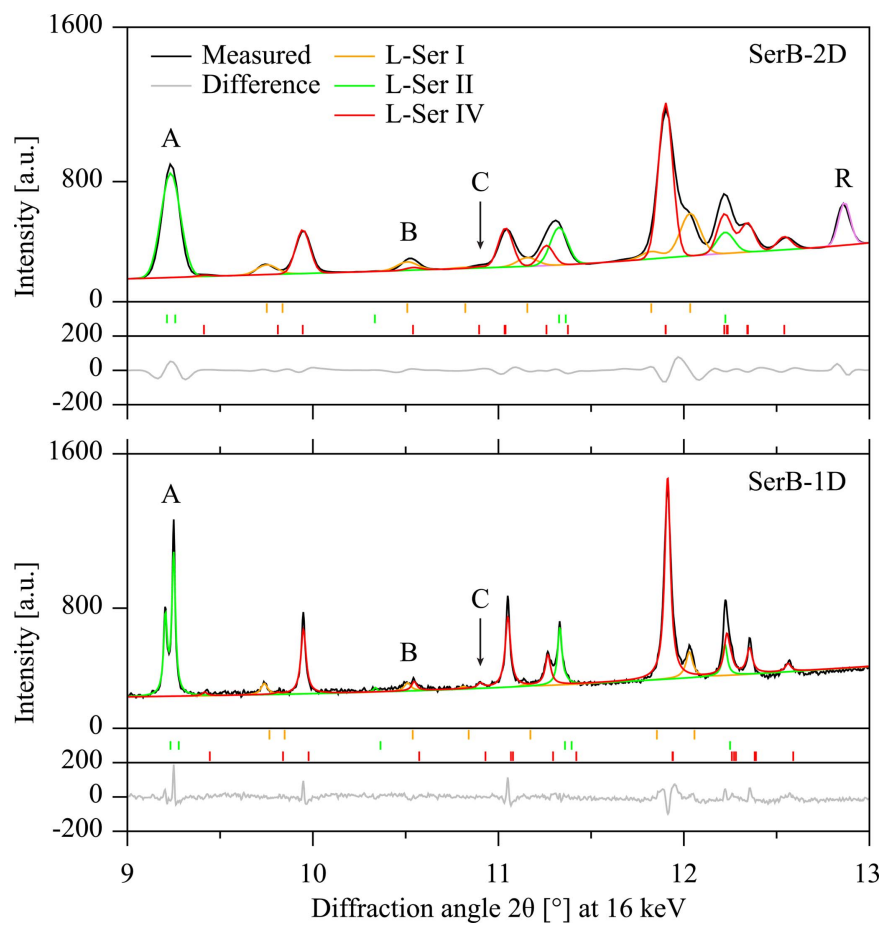

Figure 6

Enlarged regions of the Pawley refinements of samples SerB-2D (top) and SerB-1D (bottom). HKL ticks are shown below the diffraction patterns. The much better peak resolution of the one-dimensional data becomes instantly visible. The details 'A', 'B' and ' $\mathrm{C}$ ' are discussed in the text and the ruby peak in the two-dimensional data is marked with ' $R$ '. 

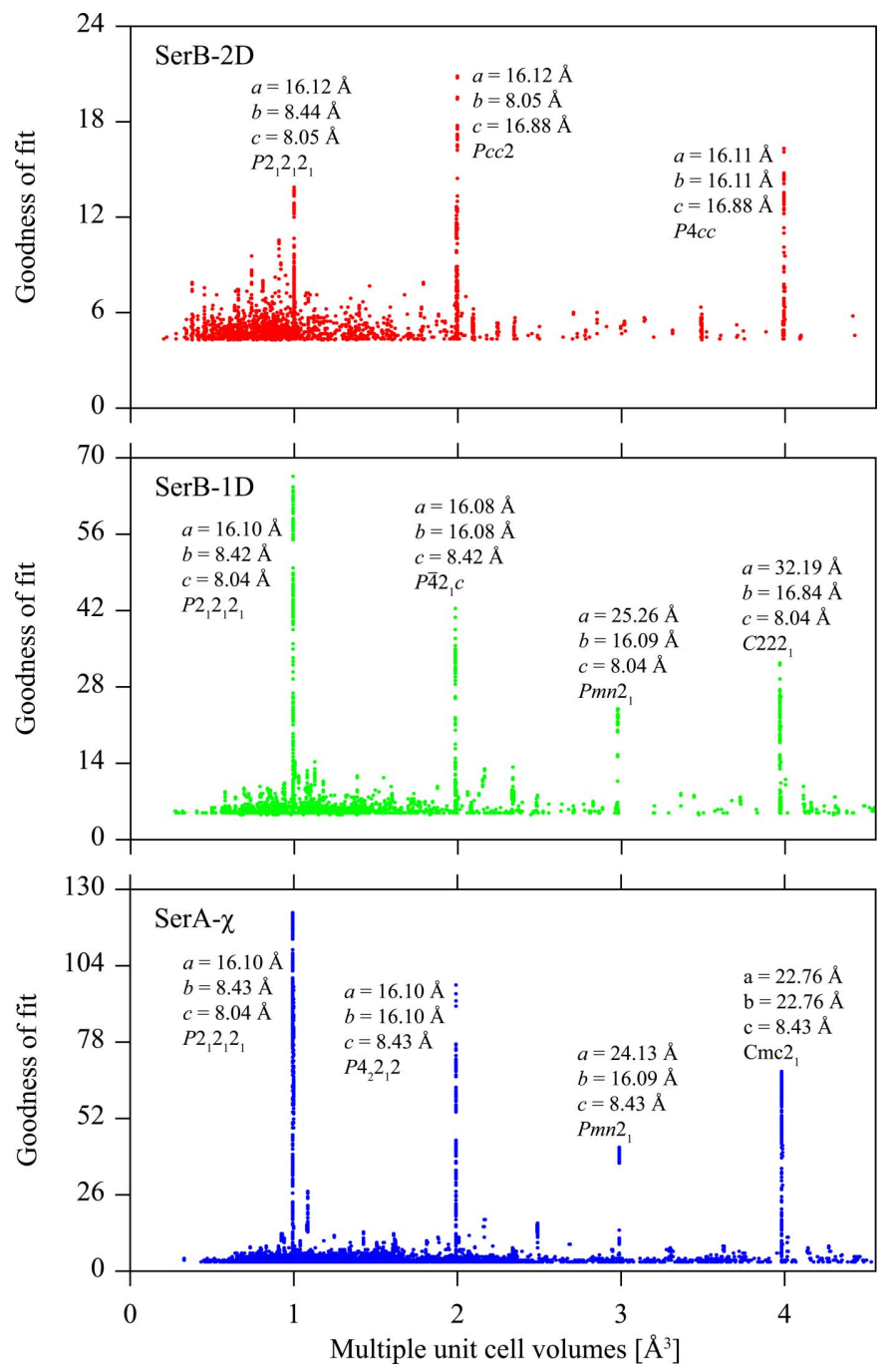

Figure 7

Goodness of fit of indexing solutions for the 25 reflections extracted from the SerB-2D (red) data, the 35 found in the SerB-1D data (green) and the 51 from the SerA- $\chi$ data (blue) plotted against multiples of the unit-cell volume. For each histogram, lattice parameters and space groups of the best solution are indicated. Structural considerations enable one to screen out non-chiral space groups for L-serine, but this plot gives a more comprehensive overview of the problems associated with each data set. dimensional data is much more reliable: the value of the $\mathrm{L}-\mathrm{Ser}$ IV peak at $10.93^{\circ} 2 \theta$ (' $\mathrm{C}$ ') is significant in the one-dimensional data ( $>5 \times$ its s.u.), whereas it is smaller than its s.u. in the twodimensional data. Moreover, even weak intensities are clearly identifiable, which results in a larger number of observed peaks in the SerA- $\chi$ data. In particular indexing of the relatively large unit cell of L-Ser IV would have been much more difficult from the SerB-2D data. From this powder pattern, 25 L-Ser IV reflections can be used for indexing, whereas 35 or 51 can be found in the SerB-1D or the SerA- $\chi$ data, respectively. When applying TOPAS's indexing method (Coelho, 2003) for all seven Bravais lattice types to the two one-dimensional data sets, the three solutions with the highest goodness of fit yield the correct lattice parameters and space group, followed by other orthorhombic, tetragonal or monoclinic space groups containing a subset of the helical symmetry of the correct space group. In contrast, from the 25 reflections found in the SerB-2D data, only unit cells with two, four and eight times larger unit cells are among the best solutions (Fig. 7). Structural considerations enable one to screen out non-chiral space groups for L-serine, but such information may not always be available. From these data, the correct solution is also found but with a much smaller goodness of fit.

Extracted intensities of L-Ser IV can be better visualized by comparing simulated powder patterns based on normalized structure factors extracted from SerA- $\chi$ and SerB-2D, as shown in Fig. 8. The largest differences are above $16^{\circ} 2 \theta$, which is the range in which the Debye ring coverage of the SerA- $\chi$ data has dropped below 100\%. Moreover and unfortunately, the two samples do not contain the same relative amounts of L-Ser II and IV. The reason is that it is almost impossible to maintain exactly the same pressure increase rate during the compression of the DACs, which is a crucial factor for the formation of the different polymorphs, even when the same final pressure is reached (Fisch et al., 2015). Rietveld quantitative phase analyses yielded $10 \mathrm{wt} \% \mathrm{~L}-\mathrm{Ser} \mathrm{I}, 12 \mathrm{wt} \% \mathrm{~L}-\mathrm{Ser}$ II and $78 \mathrm{wt} \%$ L-Ser IV for sample SerA- $\chi$, and 8, 38 and $54 \mathrm{wt} \%$, respectively, for sample SerB-2D.

The severe peak overlap of polymorphs L-Ser I and II complicates the extraction of L-Ser IV structure factors. With

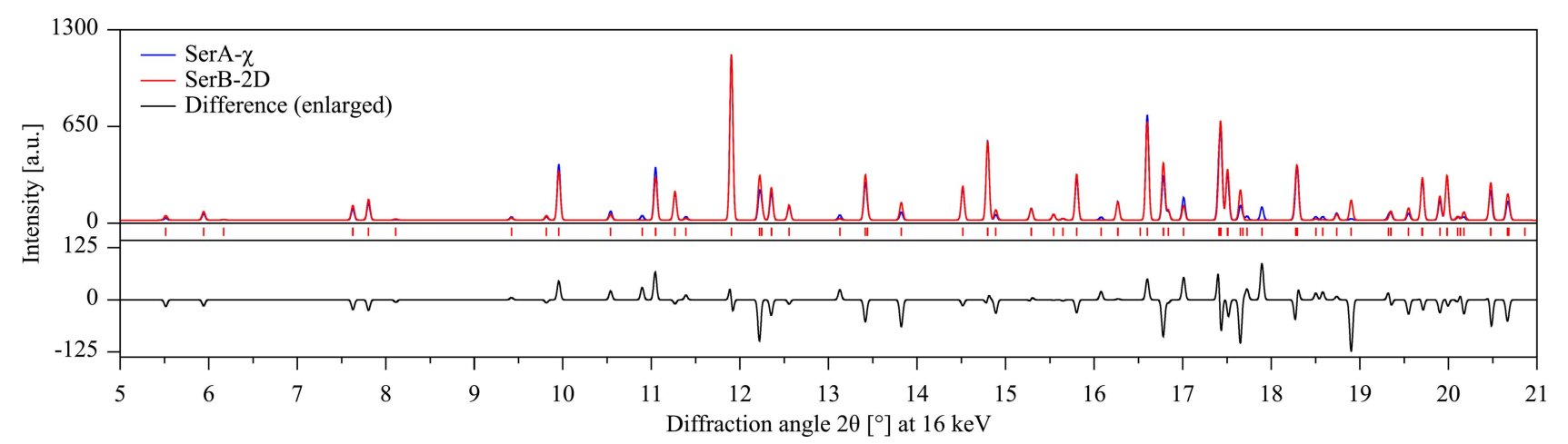

Figure 8

Calculated powder patterns based on the extracted structure factors of the SerA- $\chi$ and the SerB-2D samples. The enlarged difference curve represents $I_{\text {SerA- } \chi}-I_{\text {SerB-2D. }}$. 


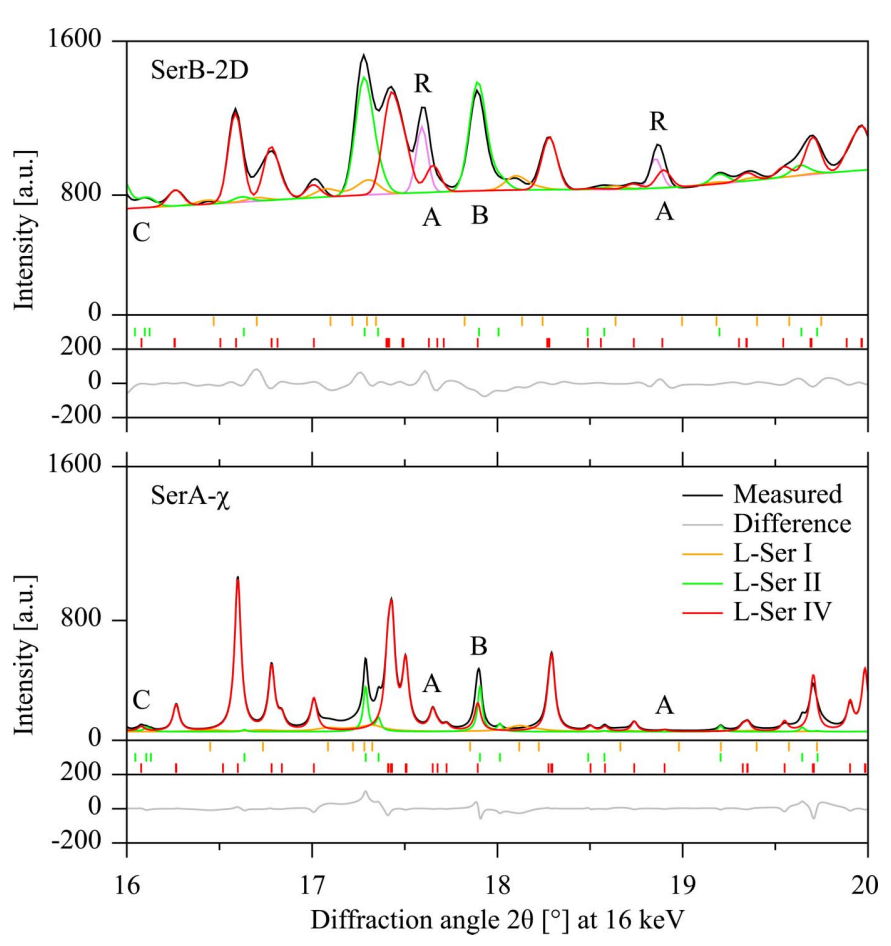

Figure 9

Enlarged regions of the combined Rietveld/Pawley refined patterns of sample SerB-2D (top) and SerA- $\chi$ (bottom). The details 'A', 'B' and 'C' are discussed in the text and the ruby peaks in the two-dimensional data are marked with ' $R$ '.

the approach of a combined Rietveld (L-Ser I and II) and Pawley (IV) refinement, contributions of overlapped peaks of L-Ser I and II can be subtracted from the data, but this approach is of course only as good as the fit of the structural models of L-Ser I at 4.5 GPa and II at $6.3 \mathrm{GPa}$ to the experimental data at the given pressure.

A preferred orientation correction on L-Ser II (210) improved the refinement significantly [March Dollase parameter of 0.885 (5) for SerB-2D and 0.862 (5) for SerA- $\chi$ ]. In both data sets, L-Ser I also seems to suffer from preferred orientation; however, no correction was applied because the broad peaks are poorly defined and the chosen structural model is most likely slightly different from the one at the actual pressure. To discuss some of the differences in more detail, enlarged regions of the combined Rietveld (L-Ser I and II) and Pawley (IV) refined raw data are shown in Fig. 9.

Precise extraction of L-Ser IV intensities is clearly disturbed by the two strong ruby peaks ('A', Fig. 9) contributing to the pattern of SerB-2D. In the one-dimensional data, the corresponding L-Ser IV peaks are well separated from those of the other phases. Another discrepancy in the extracted intensities is found at $17.9^{\circ} 2 \theta$ (' $\mathrm{B}$ '). In the SerB-2D data, the measured peak is fully explained by a broad L-Ser II reflection, whereas in the sharper SerA- $\chi$ data, a significant amount of the peak area is also accounted for by L-Ser IV. The same feature can be observed at circa $16.1^{\circ} 2 \theta$ ('C'). Even though the samples do not contain the same phase composition and, moreover, the coverage of Debye rings drops below $100 \%$ in the SerA- $\chi$ data, the differences in intensity extraction are most likely due
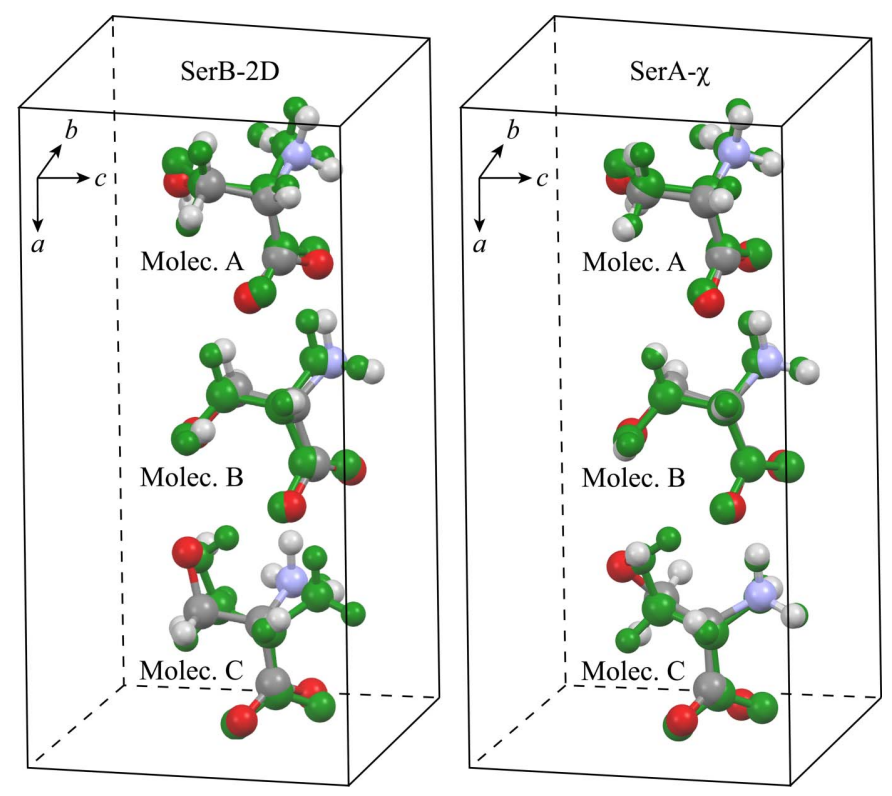

Figure 10

Asymmetric units of the final structures obtained from L-Ser IV intensities extracted from samples SerB-2D (left) and SerA- $\chi$ (right). The DFT-calculated structure (green) is shown as reference. The root mean square distance to the DFT structure increases from $0.35 \AA$ (SerA-

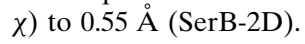

to the different peak resolutions. It is therefore unambiguous that the approach of intensity extraction from a multi-phase sample is much more stable when applied to the SerA- $\chi$ data.

Refining the converged structure obtained from the SerA- $\chi$ data against the SerB-2D data results in an increase of $R_{\mathrm{Bragg}}$ from 10.2 to $15.6 \%$, and the structure becomes slightly distorted, compared to the one obtained from the DFT calculation. This is especially expressed in the rotation of molecule $\mathrm{C}$ and its $-\mathrm{CH}_{2} \mathrm{OH}$ group torsion angle. A qualitative comparison of the final structure solutions and the DFT calculation is presented in Fig. 10. The root mean square of the distances between sites of the refined structures and the DFT geometry was compared using the IDEAL module implemented in $\operatorname{Win} G X$ version 2013.3 (Farrugia, 2012). This value increases from $0.35 \AA$ (SerA- $\chi$ ) to $0.55 \AA$ (SerB-2D), indicating better agreement with the DFT geometry for the structure obtained from SerA- $\chi$.

\section{Conclusions}

We have presented the differences between HP powder data obtained from a one-dimensional and two-dimensional detector. In conventional setups, the former is suitable for obtaining accurate lattice parameters (e.g. for determination of equation of states), as well as highly resolved peaks with a small instrumental contribution to peak shape. The latter, in contrast, is clearly superior for obtaining accurate intensities. A method combining both qualities, i.e. HP P-XRD data with exceptionally high angular resolution and appropriate intensity statistics, has also been presented. The strategy, involving one-dimensional measurements at several $\chi$ positions, has 
been successfully applied to a challenging structure solution problem. This method will not replace conventional HP powder diffraction setups with two-dimensional detectors, as the amount of time and effort needed to perform the experiment is definitely of great disadvantage. However, it can be superior to conventional setups for special problems, such as indexing of large unit cells and dealing with multi-phase powder mixtures, as well as for any investigation that requires high resolution, e.g. microstructural studies at HP.

\section{Acknowledgements}

We acknowledge support from the Swiss National Science Foundation (project 144534) and Dr Vincent Olieric for kindly providing straightforward access to the PXIII beamline at the SLS. MF thanks Dr Alan Coelho for fruitful discussions.

\section{References}

Ashiotis, G., Deschildre, A., Nawaz, Z., Wright, J. P., Karkoulis, D., Picca, F. E. \& Kieffer, J. (2015). J. Appl. Cryst. 48, 510-519.

Benecke, G., Wagermaier, W., Li, C., Schwartzkopf, M., Flucke, G., Hoerth, R., Zizak, I., Burghammer, M., Metwalli, E., MüllerBuschbaum, P., Trebbin, M., Förster, S., Paris, O., Roth, S. V. \& Fratzl, P. (2014). J. Appl. Cryst. 47, 1797-1803.

Bergamaschi, A., Cervellino, A., Dinapoli, R., Gozzo, F., Henrich, B., Johnson, I., Kraft, P., Mozzanica, A., Schmitt, B. \& Shi, X. (2010). J. Synchrotron Rad. 17, 653-668.

Boldyreva, E. V. (2007). Cryst. Growth Des. 7, 1662-1668.

Boldyreva, E. V., Sowa, H., Seryotkin, Yu. V., Drebushchak, T. N., Ahsbahs, H., Chernyshev, V. \& Dmitriev, V. (2006). Chem. Phys. Lett. 429, 474-478.

Broennimann, Ch., Eikenberry, E. F., Henrich, B., Horisberger, R., Huelsen, G., Pohl, E., Schmitt, B., Schulze-Briese, C., Suzuki, M., Tomizaki, T., Toyokawa, H. \& Wagner, A. (2006). J. Synchrotron Rad. 13, 120-130.

Caglioti, G., Paoletti, A. \& Ricci, F. P. (1958). Nucl. Instrum. 3, $223-$ 228.

Chandra Shekar, N. V. \& Rajan, K. G. (2001). Bull. Mater. Sci. 24, 121.

Coelho, A. A. (2000). J. Appl. Cryst. 33, 899-908.

Coelho, A. A. (2003). J. Appl. Cryst. 36, 86-95.

Coelho, A. A. (2012). TOPAS Academic. Version 5. Coelho Software, Brisbane, Australia.
Dovesi, R., Saunders, V. R., Roetti, C., Orlando, R., Zicovich-Wilson, C. M., Pascale, F., Civalleri, B., Doll, K., Harrison, N. M., Bush, I. J., D'Arco, P. \& Llunell, M. (2009). CRYSTAL09. University of Turin, Turin, Italy.

Dyadkin, V. (2015). SNBL Toolbox. Version 0.5. Swiss-Norwegian Beamlines, European Synchrotron Radiation Facility, Grenoble, France.

Evans, W. J., Yoo, C. S., Lee, G. W., Cynn, H., Lipp, M. J. \& Visbeck, K. (2007). Rev. Sci. Instrum. 78, 073904.

Farrugia, L. J. (2012). J. Appl. Cryst. 45, 849-854.

Fisch, M., Lanza, A., Boldyreva, E. V., Macchi, P. \& Casati, N. (2015). J. Phys. Chem. C, 119, 18611-18617.

Hammersley, A. P., Svensson, S. O., Hanfland, M., Fitch, A. N. \& Häusermann, D. (1996). High Pressure Res. 14, 235-248.

Heiney, P. A. (2005). IUCr Commission on Powder Diffraction Newsletter, No. 32, pp. 9-11.

Hinrichsen, B., Dinnebier, R. E. \& Jansen, M. (2008). Powder Diffraction Theory and Practice, edited by R. E. Dinnebier \& S. J. Billinge, pp. 414-438. Cambridge: RSC Publishing.

Ilavsky, J. (2012). J. Appl. Cryst. 45, 324-328.

Jamieson, J. C., Lawson, A. W. \& Nachtrieb, N. D. (1959). Rev. Sci. Instrum. 30, 1016-1019.

Lanza, A., Fiolka, C., Fisch, M., Casati, N., Skoulatos, M., Rüegg, C., Krämer, K. W. \& Macchi, P. (2014). Chem. Commun. 50, 1450414507.

Moggach, S. A., Allan, D. R., Morrison, C. A., Parsons, S. \& Sawyer, L. (2005). Acta Cryst. B61, 58-68.

Perillat, J. P. (2008). Min. Mag. 72, 683-695.

Prescher, C. \& Prakapenka, V. B. (2015). High Pressure Res. 35, $223-$ 230.

Rodriguez-Navarro, A. B. (2006). J. Appl. Cryst. 39, 905-909.

Smith, J. S. \& Desgreniers, S. (2009). J. Synchrotron Rad. 16, 83-96.

Syassen, K. (2008). High Pressure Res. 28, 75-126.

Toby, B. H. \& Von Dreele, R. B. (2013). J. Appl. Cryst. 46, 544-549.

Tschauner, O., McClure, J. \& Nicol, M. (2005). J. Synchrotron Rad. 12, 626-631.

Velisavljevic, N., Sinogeikin, S., Saavedra, R., Chellappa, R. S., Rothkirch, A., Dattelbaum, D. M., Konopkova, Z., Liermann, H. P., Bishop, M., Tsoi, G. M. \& Vohra, Y. K. (2014). J. Phys. Conf. Ser. 500, 032020.

Waltersperger, S., Olieric, V., Pradervand, C., Glettig, W., Salathe, M., Fuchs, M. R., Curtin, A., Wang, X., Ebner, S., Panepucci, E., Weinert, T., Schulze-Briese, C. \& Wang, M. (2015). J. Synchrotron Rad. 22, 895-900.

Willmott, P. R. et al. (2013). J. Synchrotron Rad. 20, 667-682.

Wood, P. A., Francis, D., Marshall, W. G., Moggach, S. A., Parsons, S., Pidcock, E. \& Rohl, A. L. (2008). CrystEngComm, 10, 1154-1166. 